\title{
Comparative Evaluation of Salivary Total Proteins in Deciduous and Permanent Dentition
}

\author{
Rahul Deshpande ${ }^{1}$, Suvarna Vinchurkar ${ }^{2}$, Shantanu Deshpande ${ }^{3}$ \\ ${ }^{1}$ Dr. D. Y. Patil Dental College and Hospital, Pimpri, Pune- 411018, India \\ Deenanath Mangeshkar Hospital, Pune- 411004, Maharashtra, India \\ SBL Centre of Sanskrit and Indological Studies, TMV, Pune. \\ ${ }^{2,3}$ Dr. D. Y. Patil Dental College and Hospital, Pimpri, Pune- 411018, India
}

\begin{abstract}
Human saliva plays a vital role in maintaining the integrity of oral tissues. Its composition changes during childhood due to maturation of the salivary glands indicating the need of age-matched controls for the clinical use of saliva as a diagnostic tool for diseases. This study aims at analyzing physiologic variability of naturally occurring total protein concentration in unstimulated whole saliva of children as a function of age. For this study unstimulated whole saliva specimens were collected from 20 healthy children equally divided into: Primary (3 - 5 years); Permanent (12 - 18yrs) dentition age groups. The samples were studied for total protein content by light chromatography coupled with mass spectrometry. The total protein content showed a linear increase with age. Thus this study establishes a correlation between age and salivary composition hence constructing a comprehensive catalogue which forms the basis for salivary total proteins with newer biochemical aids necessary for saliva to serve as a diagnostic aid.
\end{abstract}

Keywords: Saliva, total proteins, diagnostic tool, deciduous dentition, permanent dentition

\section{Introduction}

Mouth is a unique, highly complex multifactorial interface between the body and its external environment. It has greater structural and biologic complexity as compared to the other body orifices. It contains mineralized tissues (teeth) which are continuously exposed to environmental changes. Thus saliva, an oral bio-fluid is important in maintaining homeostasis in the oral cavity and its presence is vital to the maintenance of healthy oral tissues [1].

Saliva is composed of organic, inorganic contents and macromolecules. Salivary composition changes during childhood due to maturation of salivary glands. Thus for saliva to serve as a diagnostic aid there is a need for agematched controls with physiologic levels of salivary proteins established for particular age groups[2].

The role of saliva is much broader and it can also serve as a diagnostic tool for monitoring health and disease status of an individual [3]. This aspect of saliva is a late bloomer and is now coming to the forefront. But for this approach to succeed we must understand the basic concept of salivary composition and the role of its constituents [1].

There are only few studies on salivary composition ofhealthy children which are available [4]. Thus this study aims at analyzing the physiologic variability of naturally occurring total protein concentration in unstimulated whole saliva of children as a function of age.

\section{Review of Literature}

1] Arunkumar S et al $[2014]^{5}$

Carried out a comprehensive review on Development in diagnostic applications of saliva in oral and systemic diseases, they concluded about various advantages of a saliva as a diagnostic aid. They also defined various salivary biomarkers such as proteomic, genomic and microbial and their specific presence in various normal and abnormal conditions. They found saliva can be a diagnostic aid in various conditions like autoimmune diseases, systemic disease, hereditary diseases, malignancies and various infectious diseases. They also concluded that in the coming future, there are rich possibilities that salivary diagnostics can not only be used as a powerful tool for saving life but also to preserve those, which already have been saved.

\section{2] Devi TJ et al(2014) ${ }^{6}$}

Saliva an important physiologic fluid, containing a highly complex mixture of substances is rapidly gaining popularity as a diagnostic tool. Saliva forms a thin film, the velocity of which varies greatly at different sites and thus protects against dental caries, erosion, attrition, abrasion, periodontal diseases, candidiasis and the abrasive mucosal lesions. Early diagnosis and management of oral diseases reduces the severity and possible complications of the disease process. The use of saliva has provided a substantial addition to the diagnostic armamentarium as an investigative tool for disease processes and disorders. Its advantages as a diagnostic tool include its ease of procurement and the positive correlation between many parameters in the serum.

\section{3] Wetie A et al [2015]}

Carried out a pilot proteomic analysis of salivary biomarkers in autism spectrum disorder. The study included 12 children between the age group of 6 to 16 years old, divided into 2 sub-groups Healthy and Autistic comprising of 6 subjects each. Un-stimulated saliva was collected and analyzed for salivary proteome by using nano liquid chromatographytandem mass spectrometry. This study concluded that, nine proteins levels were significantly elevated in the saliva of children having ASD, and three protein, levels were lower or absent in same group. 


\section{International Journal of Science and Research (IJSR) \\ ISSN (Online): 2319-7064}

Index Copernicus Value (2013): 6.14 | Impact Factor (2014): 5.611

\section{4]Deshpande RR et al [2014]}

Study was carried on Total Salivary Proteins in Leukemic and Healthy Children of Mixed Dentition Age Group. The study included 9 healthy male children and 9 leukemic male children. Un-stimulated saliva was collected and analysed for total salivary proteins by using light chromatography coupled with mass spectrometry (LCMS). Sodium, potassium and calcium analysis also measured using shimadzu atomic absorption spectroscopy. Our team found markable increase of calcium levels in leukemic patient when compared with a healthy child which can also be used as a indicator in diagnosis with the help of saliva. This study concludes that there is increase level of total salivary proteins in leukemic children than healthy children.

\section{5] Deshpande $\operatorname{RR}(2015)^{9}$}

Human saliva plays a vital role in maintaining the integrity of oral tissues and its composition changes during childhood due to maturation of the salivary glands thus indicating the need of age-matched controls for the clinical use of saliva as a diagnostic tool for diseases. Thus this study aimed at analyzing physiologic variability of naturally occurring total protein concentration in unstimulated whole saliva of children as a function of age. For this study unstimulated whole saliva specimens were collected from 20 healthy children equally divided into: Primary ( 3 - 5 years); Mixed $(6-11 \mathrm{yrs})$. The samples were studied for total protein content by light chromatography coupled with mass spectrometry. The total protein content showed a linear increase with age. Thus this study establishes a correlation between age and salivary composition. Hence constructing a comprehensive catalogue which is physiologic for salivary total proteins with newer biochemical aids is necessary for saliva to serve as a diagnostic aid.

\section{Material and Methods}

\section{Criteria for Patient Selection}

In the present study, 20 normal healthy children ranging from 3 to 18 years were selected from housing societies in and around Pimpri- Chinchwad area of Pune district who were free from any systemic or local diseases which affect salivary secretions and totally caries free with $\mathrm{dmft} / \mathrm{DMFT}$ score of 0 [10] in 2015. After assessing and confirming their caries status these children were stratified equally into two dentition groups: Primary (10 children ranging from 3-5 years), Permanent (10 children ranging from 12-18years). Exclusion criteria included patients who were physical or mentally compromised, having developmental delay, auditory or visual dysfunction, known neurological diseases, history of drug intake and patients with arrested carious lesions[11].Informed consent formswere obtained from the custodial parentor guardian of the subject after explaining the procedure to the parent or guardian.

\section{Method of Saliva Collection}

To minimize the effect of circadian rhythms, all whole saliva samples were collected one hour after lunch for the unstimulated condition [4]. The child was seated in a wellventilated and well-lit room. The head was kept at 45 degrees flexion with one hand holding onto a $4 \mathrm{ml}$ cryoprecipitation vial with a funnel inserted into it, in a calm atmosphere to simulate unstimulated conditions. The saliva was allowed to drip into the funnel held to the lower lip. For each trial, the collection continued for 2 minutes but if the saliva sample was insufficient within 2 minutes, the collection was continued until $2 \mathrm{ml}$ of saliva per subject was obtained[11].

\section{Methods of Laboratory Analysis}

For detection of total proteins in saliva, the saliva samples obtained from each subject were diluted with distilled water in a proportion of 1:4.This diluted saliva sample was then subjected to inductively coupled plasma emission spectroscopy for detection of total proteins, light chromatography coupled with mass spectrometry (LCMS) was used. Mass spectrometry (MS) is an analytical technique used for determining masses of particles, for determining the elemental composition of a sample or molecule and for elucidating the chemical structures of molecules, such as peptides and other chemical compounds.

\section{Results}

Table 1: Salivary total protein in deciduous and permanent dentition

\begin{tabular}{|c|c|c|}
\hline Sr No. & Deciduous dentition & Permanent dentition \\
\hline 1. & 78.0 & 21.4 \\
\hline 2. & 48.0 & 20.2 \\
\hline 3. & 0.24 & 21.4 \\
\hline 4. & 21.30 & 26.3 \\
\hline 5. & 48.50 & 22.1 \\
\hline 6. & 40.0 & 50 \\
\hline 7. & 25.60 & 33.5 \\
\hline 8. & 49.20 & 51 \\
\hline 9. & 23.50 & 78.06 \\
\hline 10. & 0.15 & 44 \\
\hline
\end{tabular}

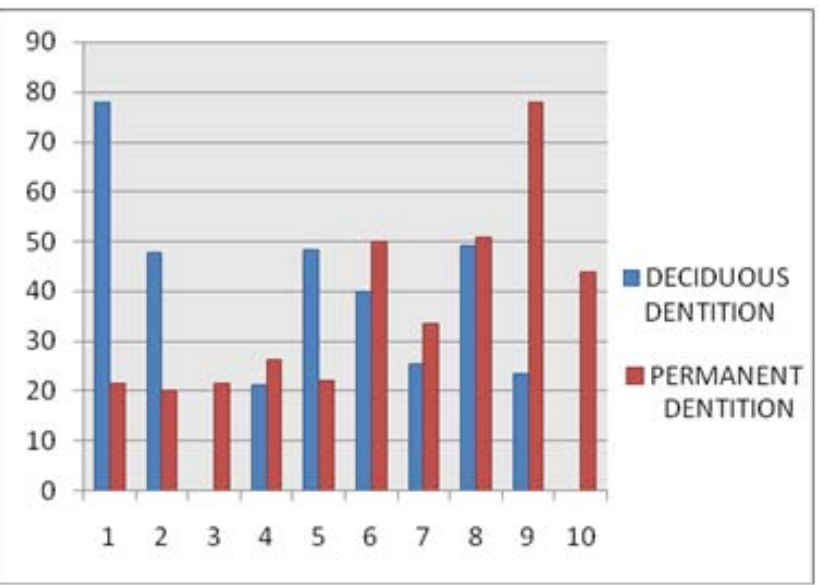

Figure 2: Bar graph showing salivary total proteins values in deciduous and permanent dentition.

The above mentioned results have been statistically analyzed by Mann Whitney test (not significant).

\section{Discussion}

The organic and inorganic contents of whole saliva were analysed in this study. A number of physiological factors influence the composition of whole saliva. These are the source of saliva, the method of collection and the degree of stimulation. Because it is difficult to use a

\section{Volume 4 Issue 12, December 2015}




\section{International Journal of Science and Research (IJSR) \\ ISSN (Online): 2319-7064 \\ Index Copernicus Value (2013): 6.14 | Impact Factor (2014): 5.611}

collecting device with children unstimulated whole saliva was collected in this research. The time of saliva collection is also important. In this study saliva was collected during acrophase as salivary flow rate peaks during afternoon time.[8]

Total proteins in saliva may have both protective and detrimental properties[9]. Thus salivary proteins can be known as double-edged swords". Function of total proteins may depend on molecule's location or site of action. Some proteins such as antimicrobial and $\mathrm{pH}$ modulating proteins play a protective role in the oral cavity, while adhesions and agglutinins play a detrimental role by increasing the colonization of microorganisms. Thus quantitative and qualitative identification of salivary proteins is a necessary first step in identifying potential protein biomarkers of disease[10]. In this study the total protein concentration showed a linear increase with age, but qualitative analysis of these proteins is essential to prove its role in health and disease.

\section{Conclusion}

From this study we can conclude that saliva has a great potential for clinical disease diagnostics. It has long been recognized that saliva serves as a mirror of body's health as it contains proteins that are frequently measured in standard blood tests to monitor health and disease[11]. Thus there is a necessity for constructing a comprehensive catalogue which is physiologic for salivary total proteins along with the qualitative analysis of individual amino acids, their linkages and formations with newer biochemical approaches. Thus this study lays a foothold and may serve as a reference value for growing interest in saliva as a diagnostic tool.

\section{References}

[1] Hay, D. I., \& Bowen, W. H. (1996). The functions of salivary proteins. Saliva and oral health, 2, 105-22.

[2] Ben-Aryeh, H., Fisher, M., Szargel, R., \&Laufer, D. (1990). Composition of whole unstimulated saliva of healthy children: changes with age. Archives of Oral biology, 35(11), 929-931.

[3] Wong, D. T. (2006). Salivary diagnostics powered by nanotechnologies, proteomics and genomics. The Journal of the American Dental Association,137(3), 313-321.

[4] BetulKargul, AysenYarat, IlknurTanboga, The Saudi Dental Journal, 1998, 10(3), 100-106

[5] Arunkumar, S., JS, A., \&Burde, K. N. (2014). Developments in diagnostic applications of saliva in oral and systemic diseases-A comprehensive review. Journal of Scientific and Innovative Research, 3(3), 372-387.

[6] Devi, T. J. (2014). Saliva - a potential diagnostic tool. IOSR Journal of Dental and Medical Sciences, 13, 52-57.

[7] Wetie, N., Armand, G., Wormwood, K. L., Russell, S., Ryan, J. P., Darie, C. C., \& Woods, A. G. (2015). A pilot proteomic analysis of salivary biomarkers in autism spectrum disorder. Autism Research.
[8] Deshpande R., Jadhav M., Patil V.(2014) Comparative Evaluation of Total Salivary Proteins in Leukemic and Healthy Children of Mixed Dentition Age Group. RJPBCS ;5(1) 126-130

[9] Deshpande R.(2015). Comparative evaluation of salivary total proteins in deciduous and mixed dentition

[10] Vieira, A. R., Marazita, M. L., \& Goldstein-McHenry, T. (2008). Genome-wide scan finds suggestive caries loci. Journal of Dental Research, 87(5), 435-439.

[11] Wu, K. P., Ke, J. Y., Chung, C. Y., Chen, C. L., Hwang, T. L., Chou, M. Y., ... \& Lee, Y. C. (2008). Relationship between unstimulated salivary flow rate and saliva composition of healthy children in Taiwan. Chang Gung Med J, 31(3), 281-6.

\section{Author Profile}

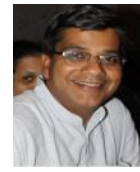

Rahul Deshpande is Professor, Dr. D.Y.Patil Dental College and Hospital, Deenanath Mangeshkar Hospital, Pune- 411004, Maharashtra, India. He is also associated with SBL Centre of Sanskrit and Indological Studies, TMV, Pune.

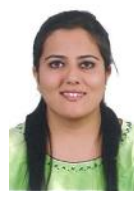

SuvarnaVinchurkar-Post graduate student, Dr. D.Y.Patil Dental College and Hospital

ShantanuDeshpande- Post graduate student, Dr. D.Y.Patil Dental College and Hospital 